\title{
A Framework for the Description of Spatiotemporal Relationships
}

\author{
Peiquan $\mathrm{Jin}^{1}$ Lihua Yue $^{1}$ \\ ${ }^{1}$ Department of Computer Science and Technology, \\ University of Science and Technology of China, 230027, Hefei, China
}

\begin{abstract}
Spatiotemporal data model, which is the basis of spatiotemporal information management, deals with the representation and manipulation of spatiotemporal data. So far many spatiotemporal data models have been proposed, but most of them concentrated on the representation of spatiotemporal objects and few of them make systematic studies on spatiotemporal relationships. Based on the semantics of spatiotemporal changes, this paper first proposes a systematic classification on spatiotemporal relationships. And then a framework for the representation of different types of spatiotemporal relationships is presented. We classify spatiotemporal relationships into two categories, which are static spatiotemporal relationships and dynamic spatiotemporal relationships. The latter is further divided into two types: time-varying topology and history topology. The definition and representation of these spatiotemporal relationships are discussed in detail. Through the research on spatiotemporal relationships, we can further develop a more functional spatiotemporal data model that support both spatiotemporal objects and spatiotemporal relationships.
\end{abstract}

Keywords: Spatiotemporal Database; Spatiotemporal Relationship; Topology .

\section{Introduction}

Spatiotemporal data management has become a critical technology for important applications such as Geographic Information Systems (GIS), environmental information systems, and multimedia. Spatiotemporal data model, which is the basis of spatiotemporal data management, deals with the representation and manipulation of spatiotemporal data. So far many spatiotemporal data models have been proposed [1-9], but most of them concentrated on the representation of spatiotemporal objects and few of them make systematic study on spatiotemporal relationships. To define a data model, not only the representation of objects but also the relationships of objects should be concerned [10]. So the representation of spatiotemporal relationships is really a key issue in spatiotemporal modeling. Traditional spatial relationships usually refer to static topological relationships among spatial objects at current time point [11-14]. But spatiotemporal relationships are far beyond this.

Based on the semantics of spatiotemporal changes in the real world, a systematic classification on spatiotemporal relationships is proposed in this paper, as well as a framework to represent different types of spatiotemporal relationships. We classify spatiotemporal relationships into two categories, which are static spatiotemporal relationships and dynamic spatiotemporal relationships. The latter is further divided into two types: time-varying topology and history topology.

\section{Spatiotemporal Changes}

One limitation that researchers of spatiotemporal databases and temporal GIS seem to impose on their models is that objects can only be created, changed and eventually removed. However this is a too simplistic view in a spatial context. Spatial objects may also split into two or more objects, and two or more objects may also be merged into a single one. There are different kinds of changes existed in the real world, and current spatiotemporal data models are usually short in supporting different types of changes completely. This is mainly because of the insufficient cognition to the real world.

According to the object-oriented view, the objects in the real world are identified by identifiers, and the state of an object is represented by its internal attributes, which consist of spatial attributes and nonspatial attributes. The former describe the position and

\footnotetext{
* This work was partially supported by the National Science Foundation of China under the grant number 60403020.
} 
region occupied by an object, and the latter are those attributes that are related to the applications an object is involved in, which are called thematic attributes. So according to the structure of an object, the spatiotemporal changes can be divided into two categories, which are: (1) life (the appearance and disappearance, and merging and splitting of objects) and (2) processes (changes of internal attributes). And processes can be further classified into spatial processes and thematic processes, according to what part of a spatiotemporal object changes.

When we consider the spatial relationships among geo-objects, we find that the spatial topological relationships among objects also change with time. This is mainly because the spatial processes of objects. E.g. as an object moves, the spatial topological relationship with other specific objects will change. Thus we define this type of change as topological change.

Thus, the spatiotemporal change of an object now could be any one among the following types:

(1) TYPE 1 (spatial processes): the spatial attributes of an object change with time, such as spread of fire, flowing of flood and change of land boundary.

(2) TYPE 2 (thematic processes): the thematic attributes of an object change with time, such as changes of soil type and change of the ownership of a land.

(3) TYPE 3 (life): changes that result in the appearance, disappearance, merging or splitting of objects

(4) TYPE 4 (topological changes): changes of an object's spatial topological relationships with other certain object. For example, the spatial topological relationship between a moving car and a road will change with time.

\section{Classification on Spatiotemporal Relationships}

Based on the study on spatiotemporal changes, we classify spatiotemporal relationships into static spatiotemporal relationships and dynamic spatiotemporal relationships, as shown in Fig.1.

The static spatiotemporal relationship between two spatiotemporal objects, which is at a certain time instant, refers to the spatial topological relationship between these two objects, E.g. in Fig.2 the spatial topological relationship between $A$ and $B$ at $t_{0}$.

Dynamic spatiotemporal relationships refer to the relationships of spatiotemporal objects along the time line. This is mainly because a spatiotemporal object has life and topological changes. We classify dynamic spatiotemporal relationships into two types: history topology and time-varying topology. History topology is the spatiotemporal relationship between a spatiotemporal object and its "parents and children", which means where and how a spatiotemporal object comes from, and what it changes into. Time-varying topology is the changing history of spatial topological relationship between two spatiotemporal objects. In Fig.2 the relationship between $A$ and $C$ along the time line is history topology, while the relationship between $B$ and $D$ from $t_{1}$ to $t_{2}$ is time-varying topology.

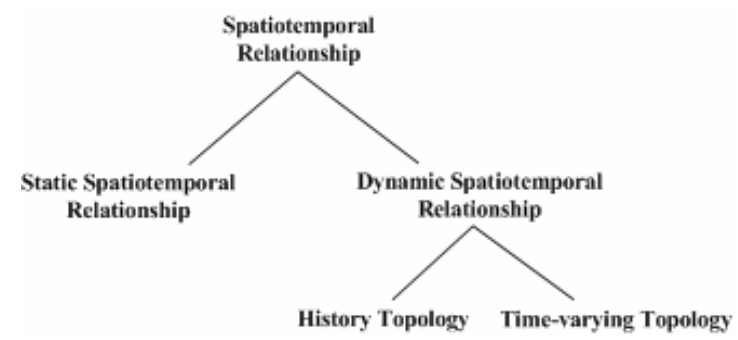

Fig. 1. Classification on spatiotemporal relationships

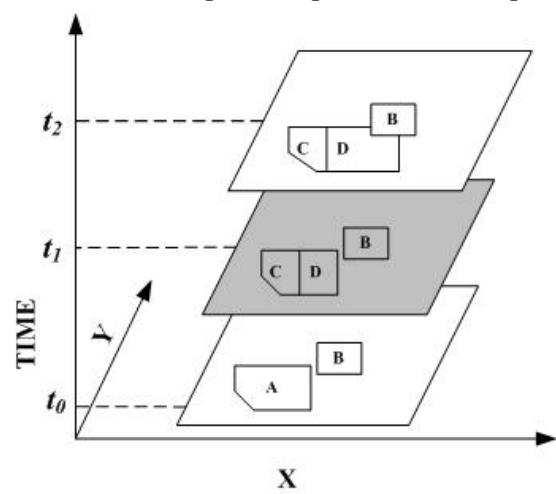

Fig. 2. Static spatiotemporal relationships and dynamic spatiotemporal relationships

\section{Representation of Spatiotemporal Relationships}

\subsection{Static Spatiotemporal Relationship}

Static spatiotemporal relationships refer to the spatial topological relationships between two spatiotemporal objects at one specific time instant. In this paper we use an implicit way to represent static spatiotemporal relationships. In spatiotemporal applications, there must be some spatial topological relationship between two different spatiotemporal objects at a given time instant, since each spatiotemporal object occupies some location in space. And more importantly this relationship may change with time. If we explicitly store the static spatiotemporal relationships in database, then we need to add new records whenever the related objects 
change. For those interested objects we can use timevarying topology to explicitly record this type of changes, but we need not and also are not able to record the static spatiotemporal relationships between any objects. In this paper, we use a set of predicates defined on spatiotemporal objects to computer static spatiotemporal relationships.

Spatial topological relationship is one of the important issues in the researches on GIS and spatial databases. So far many people studied this issue and got some valuable results [11-13], among which the 9Intersection model [11] has been widely accepted. The 9-Intersection model can completely represent spatial topological relationships. In this paper we just use the previous model to represent static spatiotemporal relationships [14].

First we define the following symbols:

Suppose that a spatial object $A$ is a point set. The boundary of $A$ is marked as $\partial A$, and the interior of $A$ is marked as $A^{\circ}$. So we can get the equation $A=\partial A \cup A^{\circ}$. And the $\operatorname{dimension}$ of $A$ is defined by $\operatorname{dim}(A)$ [14].

Then the set of predicates is defined as follows:

- Disjoint: $\operatorname{disjoint}(A, B) \Leftrightarrow A \cap B=\Phi$;

- Meet: meet $(A, B) \Leftrightarrow A^{\circ} \cap B^{\circ}=\Phi \wedge A \cap B \neq \Phi$;

- Overlap: Overlap $(A, B) \Leftrightarrow A^{\circ} \cap B^{\circ} \neq \Phi \wedge$ $\left(\operatorname{dim}\left(A^{\circ} \cap B^{\circ}\right)=\operatorname{dim}\left(A^{\circ}\right)=\operatorname{dim}\left(B^{\circ}\right)\right) \wedge A \cap B \neq$ $A \wedge A \cap B \neq B$

- Intersect: intersect $(A, B) \Leftrightarrow A^{\circ} \cap B^{\circ} \neq \Phi \wedge$ $\left(\operatorname{dim}\left(A^{\circ} \cap B^{\circ}\right)<\max \left(\operatorname{dim}\left(A^{\circ}\right), \operatorname{dim}\left(B^{\circ}\right)\right)\right) \wedge A \cap$ $B \neq A \wedge A \cap B \neq B$

- Contain: $\operatorname{contain}(A, B) \Leftrightarrow A^{\circ} \cap B^{\circ} \neq \Phi \wedge A \cap$ $B=B$;

- $\quad$ Equal: equal $(A, B) \Leftrightarrow A=B$.

Fig.3 shows an example.
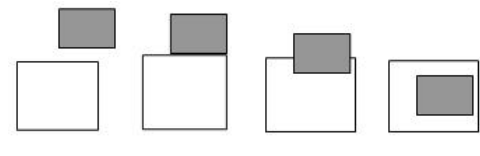

disjoint

overlap

contain

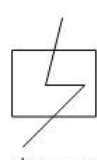

intersect

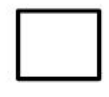

equal

Fig. 3. The set of spatial topological relationships

\subsection{Representation of History Topology}

In this paper we use an explicit way to represent history topology, which is to store each change of spatiotemporal relationships in database.

In order to define the structure of history topology, we first give the definition of history topology state.

Definition 1. history topology state: If an object $O$ appears, disappears, merges or splits at instant $t$, then the state of $O$ at $t$ is called a history topology state, denoted as $E_{h}\left(O_{p}, O_{n}, C T, t\right)$, where $O_{p}$ is the set of

object of $O$ 's ancestors and $O_{n}$ is the set of object of $O$ 's offspring, $C T$ is the type of change, it could be appearance, disappearance, merging or split.

The ancestors of $O$ denote those objects that produce $O$, while the offspring of $O$ indicate those objects that are produced from $O$.

Definition 2. history topology: The history topology $H T(O)$ of an object $O$ in $\left[t_{0}, t_{n}\right]$ is a series of history topology states $<E_{0}, E_{1}, E_{2}, \cdots, E_{m}>$, where for each $0 \leq i \leq m, E_{i}$ is a history topology state. The history topology instant of $E_{i}$ is denoted as $t_{h}{ }^{i}$ and satisfies: $t_{0} \leq t_{h}{ }^{i}<t_{h}^{i+1} \leq t_{n}, 1 \leq i \leq m-1$.

Fig. 4 is an example that shows the changing history of a land. This is very typical in land management system. In Fig. 3, a land $O_{1}$ was created at $t_{1}$ and saved in database, at $t_{2} O_{1}$ splited into $O_{2}$ and $\mathrm{O}_{3}$, and at $t_{3}$ the land $\mathrm{O}_{3}$ further splited into $\mathrm{O}_{4}$ and $\mathrm{O}_{5}$, and at $t_{4} \mathrm{O}_{2}$ and $\mathrm{O}_{4}$ were merged and became a new field $O_{6}$, and at $t_{5} O_{5}$ was eliminated from the database. The spatiotemporal relationships among these objects during the time period are represented using history topology as following:

$$
H T\left(O_{1}\right)=<\left(\Phi, \Phi \text {, appearance, } t_{1}\right),\left(\Phi,\left\{O_{2}, O_{3}\right\},\right.
$$
split, $\left.t_{2}\right)>$

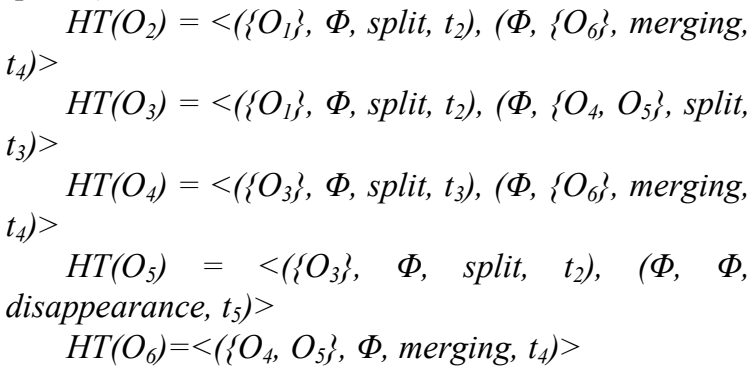

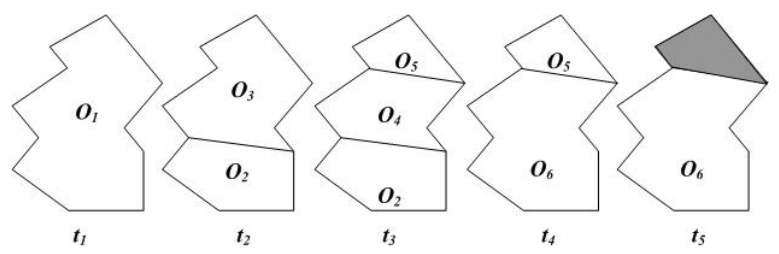

Fig. 4. History topology among lands

\subsection{Representation of Time- varying Topology}

The representation of time-varying topology is like that of history topology. We also use the explicit way. That is mainly because in most applications users are only interested in changes of spatial topological relationships between specific objects. So we must indicate of which objects we want to model the changes of spatial topological relationships.

The definition of time-varying topology is based on the spatial topological state. 
Definition 3. spatial topological state: If the spatial topological relationship between an object $O$ and another object $O_{n}$ remains static in $\left[t_{s}, t_{e}\right]$, we say the object $O$ has a spatial topological state in $\left[t_{s}, t_{e}\right]$ with object $O_{n}$. We define it as $E_{s}\left(O_{n}, T T\right.$, $\left.\left[t_{s}, t_{e}\right]\right)$, where $O_{n}$ is the object that $O$ is related with. TT is the type of spatial topological relationship, it could be disjoint, meet, overlap, contain, intersect or equal. The instant $t_{s}$ is called the start instant of $E_{s}$, while $t_{e}$ is the end instant of $E_{s}$.

Definition 4. time-varying topology: The timevarying topology $T F(O)$ of an object $O$ in $\left[t_{0}, t_{n}\right]$ is a series of spatial topological states $<E_{0}, E_{1}, E_{2}, \cdots, E_{m}$ $>$, where for each $0 \leq i \leq m, E_{i}$ is a spatial topological state. The start instant of $E_{i}$ is denoted as $t_{s}^{i}$ and the end instant of $E_{i}$ is $t_{e}^{i}$. And $T F(O)$ satisfies: $t_{s}^{0}=t_{0}$ and $t_{e}^{m}=t_{n}$ and $t_{s}^{i+1}=t_{e}^{i}+1$.

Fig. 5 is an example about time-varying topology. Suppose that in the application users want to model the changes of the spatial topological relationship between object $O$ and $O_{1}$, and then we can create a time-varying topology for the object $O$, which will record such spatiotemporal relationships. In Fig. 4 the spatiotemporal relationships between object $O$ and $O_{1}$ during the time period $\left[t_{0}, t_{3}\right]$ are represented by the time-varying topology $T F(O)$ as follows:

$T F(O)=<\left(O_{1}\right.$, disjoint, $\left.\left[t_{0}, t_{1}\right]\right),\left(O_{1}\right.$, overlap, $\left[t_{1}\right.$, $\left.\left.t_{2}\right]\right),\left(O_{1}\right.$, contain, $\left.\left[t_{2}, t_{3}\right]\right),\left(O_{1}\right.$, meet,$\left.\left[t_{3}, N O W\right]\right)>$

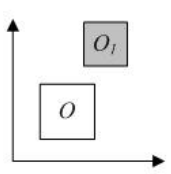

$t_{0}$

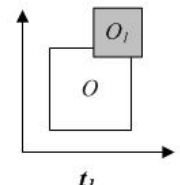

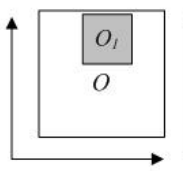

$t_{3}$

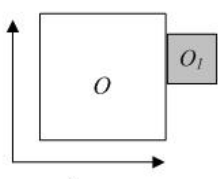

Fig. 5. Topological changes occur in $[\mathrm{t} 0, \mathrm{t} 3]$

\section{Conclusions and Future Work}

Spatiotemporal data model deals with representation and manipulation of spatiotemporal objects, as well as spatiotemporal relationships. Unfortunately, so far there is no systematic research on spatiotemporal relationships. In this paper, we have deeply studied the spatiotemporal relationships and presented a framework for the modeling of spatiotemporal relationships. The research on spatiotemporal relationships can be used to build a relatively functional spatiotemporal data model, which can represent not only spatiotemporal objects but also spatiotemporal relationships. Future work will focus on the development of a unified spatiotemporal data model that supports both spatiotemporal objects and spatiotemporal relationships.

\section{Reference}

[1] G.Langran, "Time in Geographic Information Systems", Taylor \& Francis Ltd., 1992

[2] E.Camossi, M.Bertolotto, E.Bertino, et al., "A Multigranular Spatiotemporal Data Model". Proc. of ACM-GIS, pp.94-101, 2003

[3] C.Claramunt and M.Theriault, "Toward semantics for modeling spatio-temporal processes within GIS", Proc. of SDH, pp. 47-63, 1996

[4] D.Peuquet and N.Duan, “An Event-Based Spatiotemporal Data Model for Temporal Analysis of Geographical Data", IJGIS, 1, pp.724, 1995

[5] L.Forlizzi, R.Güting, E.Nardelli, et al., "A Data Model and Data Structures for Moving Objects Databases", Proc. of SIGMOD, pp.319-330, 2000

[6] R.Güting, M.Böhlen, M.Erwig, et al.: A Foundation for Representing and Quering Moving Objects, TODS, 25(1), pp.1-42, 2000

[7] J.Peiquan, Y.Lihua and G.Yuchang, "An Extension to the ADT-based Spatiotemporal Data Model". Proc. of IKE, pp.216-221, 2004

[8] K.Wang, C.Fierbinteanu and M.Maekawa, "A Conceptual Framework for Spatiotemporal Data Modeling", Proc of DEXA, pp.57-66, 2003

[9] Y.Baolin and F.Yucai, "A Detection Model of Direction Relations for Spatial and Spatiotemporal Objects", Proc of $A P W e b$, pp.874-878, 2004

[10] A.Silberschatz, H.Korth and S.Sudarshan, "Database System Concepts", Third Edition, McGraw-Hill, 1999

[11] M.Egenhofer and J.Herring, "Categorizing Binary Topological Relations Between Regions, Lines, and Points in Geographic Databases", Technical Report, Department of Surveying Engineering, University of Maine, 1990

[12] M.Egenhofer, J.Sharma and D.Mark, "A Critical Comparison of the 4- Intersection and 9Intersection Models for Spatial Relations: Formal Analysis", Autocarto, 11, 1993

[13] M.Egenhofer and R.Franzosa, "Point Set Topological Relations”, IJGIS, 5, pp.161-174, 1991

[14] E.Clementini, P.Di Felice and P.van Oosterom, "A Small Set of Formal Topological Relationships Suitable for End-User Interaction”, Proc. of SSD, pp.277-295, 1993 